\title{
Improving Capacity of Smart Grid Wireless Backhauls with Deadline Ordered Scheduler and Packet Concatenation
}

\author{
Ting-Chu Lee and Zsehong Tsai
}

\begin{abstract}
Many applications in the smart grid generate packets with small data payload and may lead to low channel efficiency. In addition, some types of information exchanging from the smart meters to the control center is useful only within the predefined delay constraint. Hence, given that the concentrators are assigned a fixed amount of bandwidth, the maximal number of nodes that the smart grid wireless backhaul can support without violating their delay constraints becomes an important topic. In this study, we proposed to employ a deadline ordered scheduler with packet concatenation and obtained the call admission control (CAC) to resolve these two issues. The performance of this novel design were demonstrated via analytical and simulation results. It is shown that the proposed scheduler with packet concatenation significantly improves the capacity of the smart grid wireless backhaul.
\end{abstract}

Index Terms-Smart grid, wireless backhaul, deadline ordered scheduler, packet concatenation, call admission control, delay guarantees.

\section{INTRODUCTION}

Nowadays, the smart grid, next-generation grid, has attracted much attention. The most significant difference between the traditional grid and smart gird is that smart grid provides two-way flow of electrons and information which allows pervasive control and monitoring of the grid. In this paper, we consider a general model of the smart grid shown in Fig. 1. It is composed of three major parts i.e., power generation, power distribution, and power consumption [1], [2]. In almost all cases, the communication network of a smart grid, which plays the key function of interconnecting these three major parts, can be classified into three categories: home area network (HAN), neighborhood area network (NAN) and wide area network (WAN) [2], and for the convenience of deployment, wireless backhauls such as LTE [3] are often employed. In such typical deployment models, concentrators are used to concentrate smart meter traffic flows from HAN and NAN and then transport these flows via WAN back to the control center, as shown in Fig. 1.

Many researcher have noticed that many applications in the smart grid may generate small packets and may lead to low channel efficiency. To provide an efficient data transmission mechanism for short data burst transmissions from a large number of devices is a major challenge for the smart grid communications network [4].

Manuscript received March 10, 2014; revised April 30, 2014

The authors are with the Graduate Institute of Communication Engineering, National Taiwan University, Taipei, Taiwan (e-mail: tingchulee@gmail.com, ztsai@ntu.edu.tw).

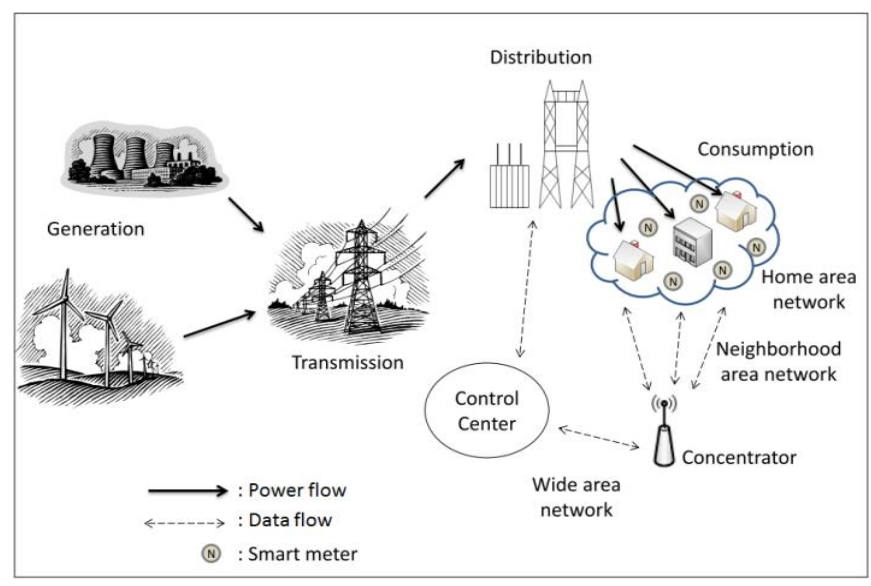

Fig. 1.A general model of the smart grid

In addition, some types of information exchanging from smart meters to the control center are useful only within the predefined delay constraint [5]. Hence, when the concentrators are assigned a fixed amount of bandwidth, the maximal number of nodes that the smart grid wireless backhaul can support without violating their delay constraints becomes an important topic.

Thus, although many important design issues are under discussions, in this paper we will focus on two network related issues in smart grid design, the high protocol overhead and the enforcement of delay guarantees, as elaborated in the following.

The high protocol overhead: In a smart grid, when there exist many applications generating packets with small data payloads, the ratio of protocol overhead become very high. The high protocol overhead may reduce the channel efficiency and should be avoided as much as possible.

Enforcement of delay guarantees: An important requirement of the smart grid communication is the strict delay requirement. If the communication delay of a particular type of information exceeds its delay constraint, the information would be no longer useful and would probably degrade the quality of the grid. In many cases, the wireless WAN bandwidth is fixed. Hence, the number of nodes that such a smart grid network can support without violating their application delay constraints becomes an important design issue.

The contribution of this research work is to address these two issues. We first propose to employ packet concatenation as the solution for the first problem. The idea of concatenating multiple packets into a single frame or packets has been found to be an effective way to increase the capacity of the wireless channel in the literature since it can reduce the protocol overhead [6], [7]. Recently, many wide area network standards also incorporate the ability to concatenate multiple IP packets into single MAC payload, such as WCDMA and 
LTE. However, the big size of IP header and UDP header compared to the small payload size can still be a significant overhead. Hence, in our work, we employ packet concatenation at the application layer (AL) to further reduce the header overhead. Secondly, when a fixed-bandwidth wireless channel is allocated to the concentrator, we develop a call admission control (CAC) procedure in order to fulfill the delay guarantee requirement in the smart grid network. In addition, a deadline ordered scheduler [8] is adopted in the concentrator in order to further increase the capacity and enlarge the call admissible region.

Many literatures have been investigated on the delay issue in smart grid. In [2], an optimal cluster formation problem using dynamic programming algorithm was used to minimize the cost due to QoS degradation from packet violating its delay constraint and packet loss. More recently, $\mathrm{Xu}$ and Wang [5] provided the delay bound from the electrical devices to the gateway in the wireless mesh network in smart grid.

Meanwhile, a vast amount of research has been conducted on both deadline ordered scheduler and packet concatenation issues. As for the deadline ordered scheduler, Figueria and Pasqueal [8] showed that there is a simple schedulability condition for all deadline-ordered service disciplines no matter how one calculates deadlines. As for packet concatenation, Wang, Liew, and $\mathrm{Li}$ [6] proposed a multiplex-multicast scheme which concatenates multiple downlink VoIP packets into one MAC layer frame to overcome the large overhead of transmitting VoIP traffic over an 802.11 WLAN. Afterwards, Hong and Tsai [7] combined the ideas of deadline ordered scheduler and packet concatenation. They derived the delay bound of the multi-channel deadline ordered scheduler which adopted packet concatenation. Based on the delay bound formula, call admission control of a broad range of scheduling algorithms were obtained. These studies inspired us to derive the call admission control region to determine the maximum number of nodes/applications to be supported in a smart grid when their concentrators employ deadline ordered scheduler and packet concatenation.

The rest of this paper is organized as follows: Section II gives the overview of the system architecture and related wireless protocols. In Section III, we give an example to show how one can use the results of [7] to execute the call admission control. Then, the performance evaluation of the proposed design by simulation will be demonstrated in Section IV. Section V draws the conclusion of the paper.

\section{System ARChitecture AND NETWORK MODEL}

\section{A. System Architecture}

The system architecture is shown in Fig. 2, where only the uplink data flow scenario is studied and described since the uplink flows are more delay sensitive. First, the data are transferred from the smart meter to the HAN gateway, which will then send the sub-packets to the concentrator via NAN. (To avoid confusion, "sub-packet" means the smart meter data payload with necessary information that would be concatenated in a WAN "packet.")Then, the concentrators forward the sub-packets to the WAN base station. Finally, the base station transmits the sub-packets to the control center. Note that a concentrator also needs to concatenate the sub-packets received from HANs.

As for the communication technology of HAN, ZigBee [9] is adopted here due to its low power consumption and simple network configuration. As for NAN, we choose PLC as the lower-layer communication technology and adopt HomePlug AV2 [10] standard. Finally, as for the communication technology of WAN, we focus on LTE since it is the most popular wireless WAN technology today, and we will employ packet concatenation with LTE at the concentrators.

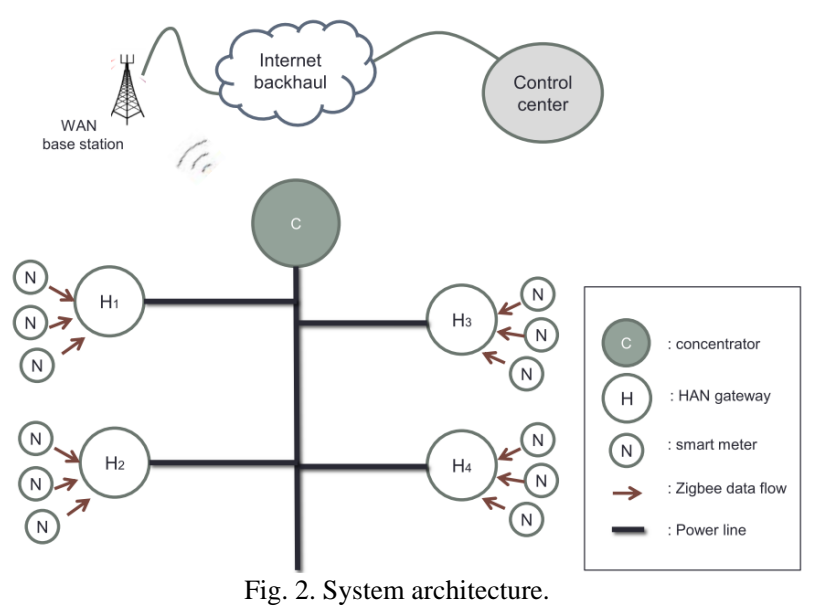

\section{B. Overview of Zigbee MAC Layer and Data Frame}

Zigbee adopts IEEE 802.15.4 as its PHY and MAC layer protocols. The IEEE 802.15.4 MAC supports two operational modes, i.e., non beacon-enabled mode and beacon-enabled mode that can be selected by the personal area network (PAN) coordinator. In our work, the beacon-enabled mode is adopted since it provides guaranteed bandwidth and bounded end-to-end delay for applications requiring low-latency.

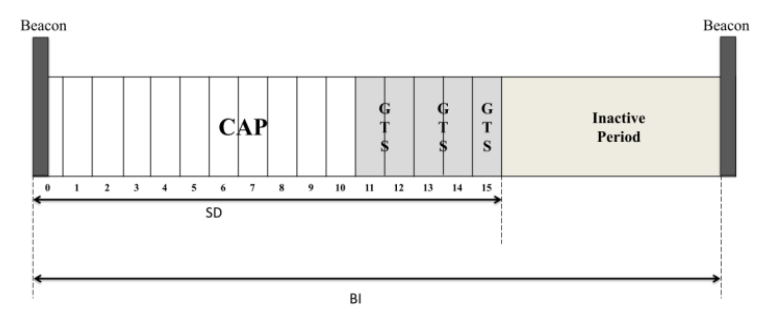

Fig. 3. Zigbeesuperframe structure.

In beacon-enabled mode, the superframe structure (see Fig. 3 ) is used to manage the communication between nodes associated to the PAN. The superframe, which is contained in a Beacon Interval (BI) bounded by two consecutive beacon frames, includes an active period and an inactive period (optional). The lengths of Superframe Duration (SD) and Beacon Interval are described by the values of BeaconOrder (BO) and SuperFrameOrder (SO), respectively [9]. The active period, which is divided into 16 equally-sized time slots, consists of contention access period (CAP) and contention free period (CFP). In CAP, nodes compete for medium access using slotted carrier sense multiple access with collision avoidance (CSMA/CA). On the other hand, the CFP contains guaranteed time slots (GTSs), which always appear at the end of the active superframe starting at a slot 
boundary immediately following the CAP. The PAN coordinator may allocate up to seven of these GTSs and a GTS can occupy more than one slot period. Here, we assume that all nodes communicate in CFP, which provide assured bandwidth and delay guarantees [11]. As for the Zigbee data frame structure, it contains a maximum of 101 byte of application payload and a total of 34 byte of headers in different layers [9].

\section{Overview of HomePlug AV2 MAC Layer}

The HomePlug AV2 MAC supports time-division multiple access (TDMA) used for applications requiring QoS guarantees and carrier-sense multiple access (CSMA) used for elastic applications. To coordinate access to the medium, each HomPlug AV2 network has a central coordinator that periodically broadcasts a beacon with information on TDMA and CSMA allocation. The Beacon period in HomePlug AV2 is set to be twice the AC line cycle and synchronized with the underlying AC line cycle. Thus, HomePlug AV2 has a beacon period of $33.33 \mathrm{~ms}$ and $40 \mathrm{~ms}$ in countries with $60 \mathrm{~Hz}$ and $50 \mathrm{~Hz} \mathrm{AC}$ line cycle frequencies, respectively. The beacon period structure is shown in Fig. 4. The MAC data plane flow is briefly reviewed as follows. The MAC receives MAC Service Data Units (MSDUs, see Fig. 5) and encapsulates them with a header, optional Arrival Time Stamp (ATS), and a checksum to create a stream of MAC frames. The stream is then divided into 512 octet segments, encrypted and encapsulated into serialized PHY Blocks, and packed as MAC Protocol Data Units (MPDUs) to the PHY unit which then generates the final PHY Protocol Data Unit (PPDU) to be transmitted onto the power line [10], [12].

Beacon Period: 33.33 ms over $60 \mathrm{~Hz}$ AC Line Cycle; $40 \mathrm{~ms}$ over $50 \mathrm{~Hz}$ AC Line Cycle

\begin{tabular}{|c|c|c|}
\hline Beacon & CSMA Allocation & TDMA Allocation \\
\hline
\end{tabular}

Fig. 4. HomePlug AV2 beacon structure.

\begin{tabular}{|l|c|c|c|c|}
\hline ODA (6) & OSA (6) & VLAN (4) & Ethertype (2) & Payload (46 1500) \\
\hline
\end{tabular}

\section{Overview of Long Term Evolution Data Plane}

Fig. 6 shows an example of uplink data flow through various protocol layers. Assume that the user equipment (UE) is already attached to the LTE network, IP packets (which contain the entire UDP and application payload) are first received by the Packet data compression protocol (PDCP) layer. PDCP performs ciphering, adds PDCP header, and then forwards the packet to the Radio Link Control (RLC) layer. RLC will concatenate and/or segment the PDCP SDUs, add an RLC header, and pass them to Medium Access Control (MAC) layer. MAC will multiplex the RLC PDUs and attach a MAC header to form a transport block, which is needed to be transmitted in $1 \mathrm{~ms}$ sub-frame. The transport block size depends on the modulation and coding scheme (MCS) and the number of resource blocks allocated to the UE [3].

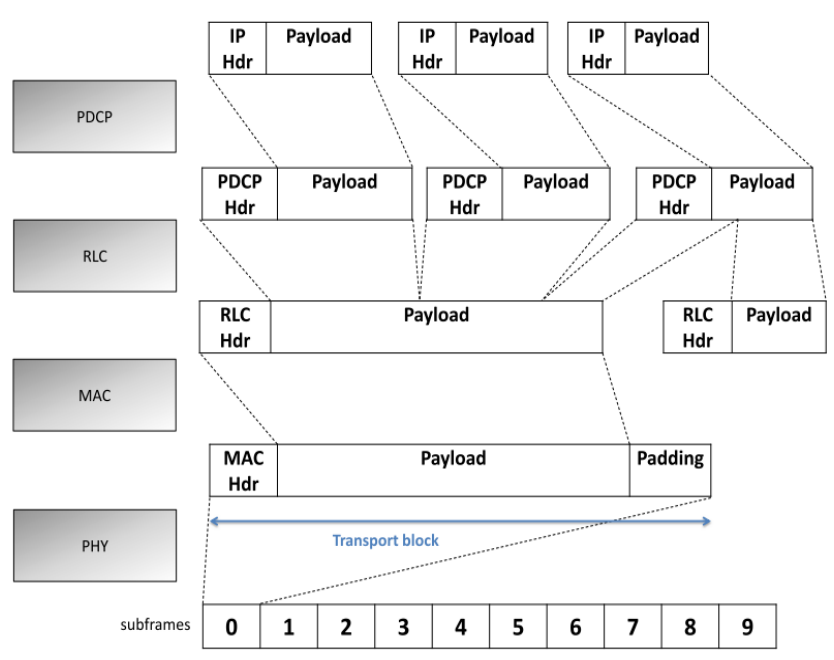

Fig. 6. LTE data plane overview.

\section{E. The Procedure of the Smart Meter Data Flow}

Finally, the procedure of the smart meter data flow through the whole system is stated as follows. First, smart meters transmit the data to the HAN gateway via Zigbee. Since the HAN gateway communicates with the concentrator using a protocol different from Zigbee, some parts of the network layer header and application service (APS) layer header are no longer needed for further transmission. The parts in networklayer header should be remained are i) source address: the control center can know where the smart meter application information is from, ii) sequence number: the control center can reconstruct all application information in order, regardless of any fragmentation, disordering, or packet loss that may occur during transmission. The parts in APS header should be remained are i) Source and Destination Endpoint: the control center can know which application the information corresponds to. In addition to removing some unnecessary parts of the header, we need to add an extra byte of length indicator such that the control center can successfully segment the received concatenated packets into correct application flows. As a result, after receiving the Zigbee data, the HAN gateway will remove the unnecessary part of the headers and add one byte length indicator to form the sub-packet, which will be the payload of the MSDU of HomePlug AV2 and transmitted to the concentrator (see Fig.7). Finally, the concentrator will concatenate all received sub-packets using Strictly Deadline-Ordered (SDO) algorithm [7] at the AL and the upper limit $L_{\max }$ on the total length of the sub-packets concatenated in an AL payload of a LTE packet can be calculated as follows,

$$
\begin{aligned}
L_{\max }=T B S & -\left(H_{U D P}^{L T E}+H_{I P \nu 6}^{L T E}+H_{P D C P}^{L T E}\right. \\
& \left.+H_{R L C}^{L T E}+H_{M A C}^{L T E}\right),
\end{aligned}
$$

where $H_{U D P}^{L T E}, H_{I P v 6}^{L T E}, H_{P D C P}^{L T E}, H_{R L C}^{L T E}$, and $H_{M A C}^{L T E}$ denotes the size of LTE UDP header, IPv6 header, PDCP header, RLC header, and MAC header, respectively, and TBSisthe size of the transport block.

\begin{tabular}{|c|c|c|c|c|c|}
\hline $\begin{array}{c}\text { Length } \\
\text { indicator (1) }\end{array}$ & $\begin{array}{c}\text { Source } \\
\text { address (2) }\end{array}$ & $\begin{array}{c}\text { Sequence } \\
\text { Number (1) }\end{array}$ & $\begin{array}{c}\text { Dest. } \\
\text { Endpoint (1) }\end{array}$ & $\begin{array}{c}\text { Source } \\
\text { endpoint (1) }\end{array}$ & Application payload \\
\hline
\end{tabular}

Fig. 7. The sub-packet structure. 


\section{CAll Admission CONTROL}

Call admission control (CAC) is the process of regulating traffic volume in smart grid network, ensuring that each authorized application flow in the network would meet its delay constraint. According to the CAC, the call admissible region should indicate the maximal number of node a smart grid network can support without violating their delay constraints. Our approach to derive CAC for the deadline ordered scheduler using Delay-EDD algorithm [13] with packet concatenation is similar to [6]. Assume that there are $I$ classes of smart meter application flow. Each of the class- $I$ flow is constrained by an affine arrival curve $\alpha_{b_{i}, r_{i}}(t)$ (please refer to [14] for details).We aim to find the parameters of the affine arrival curve $\alpha_{b_{i}^{*}, r_{i}^{*}}(t)$ by which each of the class-isub-packet flow injecting into the concentrator is constrained. Also, each class- $i$ flow is assigned a fixed delay constraint $d_{i}$ (Here delay constraint of a flow has excluded the processing delay of all hardware and software in the system and the LTE core network.)Hence, the deadline of the class- $i$ sub-packet entering the deadline ordered queue (DOQ) of concentrator is equal to the delay constraint $d_{i}$ minus the worst case delay $D_{i}^{M 2 C}$ from the smart meter to the concentrator, i.e., the sum of the worst case delay from the smart meter to the HAN gateway and from the HAN gateway to the concentrator. For example, if a sub-packet of class- $I$ flow with delay constraint $d_{\mathrm{i}}$ and the worst case delay $D_{i}^{M 2 C}$ enters the concentrator at time $\tau$, its deadline is $\tau+d_{i}-$ $D_{i}^{M 2 C}$. With these parameters, if there are $n_{i}$ class- $i$ flows, the maximum amount of traffic load fed into DOQ during [0, $\tau]$ with deadlines earlier than $\tau$ is $n_{i}\left[r_{i}^{*}\left(\tau-d_{i}^{*}\right)+b_{i}^{*}\right] \mathrm{u}\left(\tau-d_{i}^{*}\right)$ where $\mathrm{u}(t)$ is the unit step function and $d_{i}^{*}=d_{i}-D_{i}^{M 2 C}$.

The call admissible region is determined by applying the bounded appetite condition for a constant $\theta$ [7], and it consists of the following inequality:

$$
\sum_{i=1}^{I} n_{i}\left[r_{i}^{*}\left(\tau-d_{i}^{*}\right)+b_{i}^{*}\right] \mathrm{u}\left(\tau-d_{i}^{*}\right) \leq C_{e q}^{w} \tau-\theta, \forall \tau>d_{1}^{*},
$$

where $C_{e q}^{w}$, which depends on the length distribution of sub-packet, $L_{\max }$, and other parameters, is the worst case equivalent capacity for the concentrator [7]. After rearranging the inequality and some algebraic operations, we have

$$
\left\{\begin{array}{l}
\sum_{i=1}^{I} n_{i} r_{i}^{*} \leq C_{e q}^{w} . \\
\sum_{i=1}^{j} n_{i}\left[r_{i}^{*}\left(d_{j}^{*}-d_{i}^{*}\right)+b_{i}^{*}\right] \leq C_{e q}^{w} d_{j}-\theta, \quad j=1, \ldots, I .
\end{array}\right.
$$

To analytically derive the call admissible region,one should derive the delay bounds in $\operatorname{HAN}$ and NAN $\left(D_{i}^{M 2 C}\right)$ and the affine arrival curve $\alpha_{b_{i}^{*}, r_{i}^{*}}(t)$ using network calculus [14] based on the protocol parameters such as data rates, the superframe structure, and the beacon structure elaborated in Section II, assuming the worst case scenario. The detailed procedure is omitted in this paper due to lack of space.

\section{Performance Evaluation}

We first demonstrate a numerical example of CAC for two traffic classes in the smart grid network. Assume that class- $i$ flow periodically generates packets of fixed length $l_{\mathrm{i}}$ (which corresponds to sub-packets of fixed length $l_{i}^{*}$ ) with period $T_{1}$ and have a delay constraint $d_{\mathrm{i}}$, for $i=1,2$. Without loss of generality, we assume that $d_{2} \geqq d_{1}$. The parameters of the smart grid application and different channels for the following two scenarios are listed in Table I and Table II [4], [15].

TABLE I: PARAMETERS SETTING OF THE SMART GRID APPLICATION

\begin{tabular}{|l|l|}
\hline Control traffic parameters & Values \\
\hline Zigbee MAC packet length & 64 Bytes \\
Period & $1 \mathrm{sec}$ \\
Delay constraint & $100 \mathrm{~ms}$ \\
\hline Billing/Usage traffic parameters & Values \\
\hline Zigbee MAC packet length & 64 Bytes \\
Period & $2 \mathrm{sec}$ \\
Delay constraint & $150 \mathrm{~ms}$ \\
\hline Environmental sensing traffic parameters & Values \\
\hline Zigbee MAC packet length & $200 \mathrm{Bytes}$ \\
Period & $2 \mathrm{sec}$ \\
Delay constraint & $150 \mathrm{~ms}$ \\
\hline
\end{tabular}

TABLE II: PARAMETERS SETTING OF THE SMART GRID NETWORK

\begin{tabular}{|l|l|}
\hline Zigbee channel parameters & Values \\
\hline BeaconOrder (BO) & 1 \\
SuperFrameOrder (SO) & 1 \\
PHY data rate & $250 \mathrm{Kbps}$ \\
\hline HomePlug AV2 parameters & Values \\
\hline Beacon period & $33.33 \mathrm{~ms}$ \\
PHY data rate & $2 \mathrm{Gbps}$ \\
\hline LTE channel parameters & Values \\
\hline MCS index & 19 \\
\# of resource block allocated to the concentrator & 7 \\
Transport block size & 373 Bytes \\
\hline
\end{tabular}

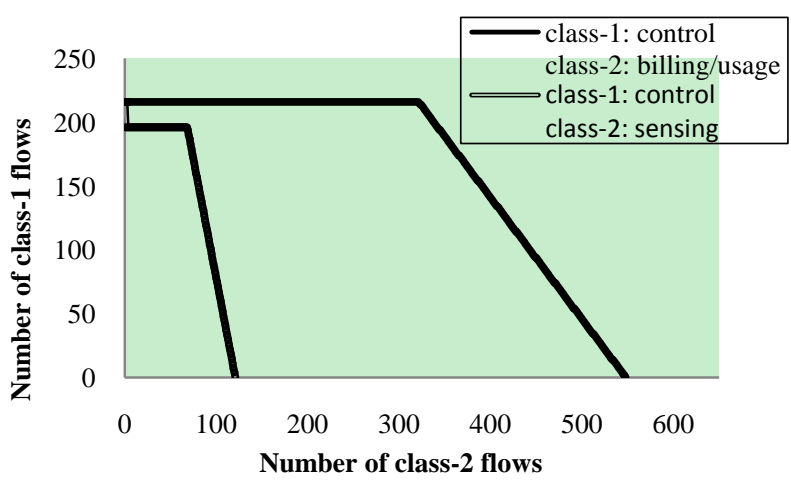

(a)

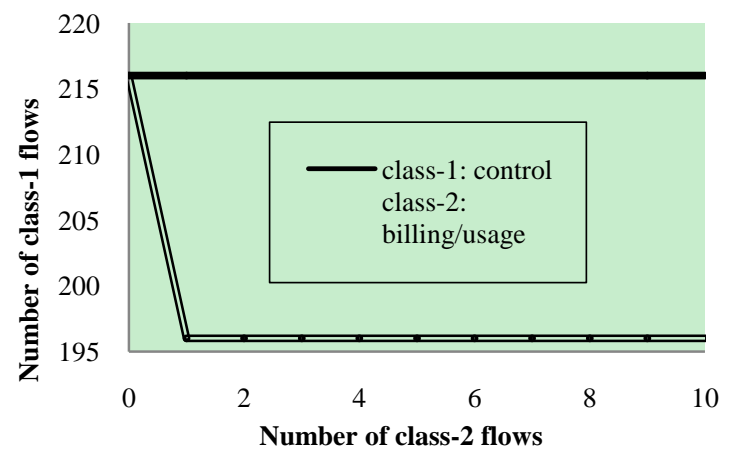

(b)

Fig. 8. (a) Analytical call admissible regions of the deadline ordered scheduler with packet concatenation. (b) a partial enlarged sectional view of the head portion of Fig. 8 (a).

Fig. 8 (a) shows the analytical results of the 
delay-bound-based call admissible regions in two scenarios, where a partial enlarged sectional view of the head portion is illustrated in Fig. 8 (b). As one can easily observe, the white curve has a jump at $n_{2}=0$, and this is because that when there are only class-1 flows, we have only fixed-length small sub-packets, and thus higher $C_{e q}^{w}$ is achieved. However, as for the black curve, the length of both class- 1 sub-packet and class-2 sub-packet are the same, $C_{e q}^{w}$ remains the same after class- 2 flows joining the network, and thus there is no jump at $n_{2}=0$. In the flat part of both curves, the number of class- 1 flows remains unchanged and are not affected by the increase of class- 2 traffic. It is because even if all class- 2 sub-packets (with larger deadline)enter the DOQ earlier than class-1 sub-packets(with smaller deadline),DOQ can place the sub-packet with larger deadline at the tail of the queue, which will not affect the sub-packet with smaller deadline. The roll-off part of both curves indicates that when the number of class-2 flows exceeds a certain value, in order to meet the deadline guarantee, the number of class-1 flow should be decreased to admit additional class- 2 flows accordingly.

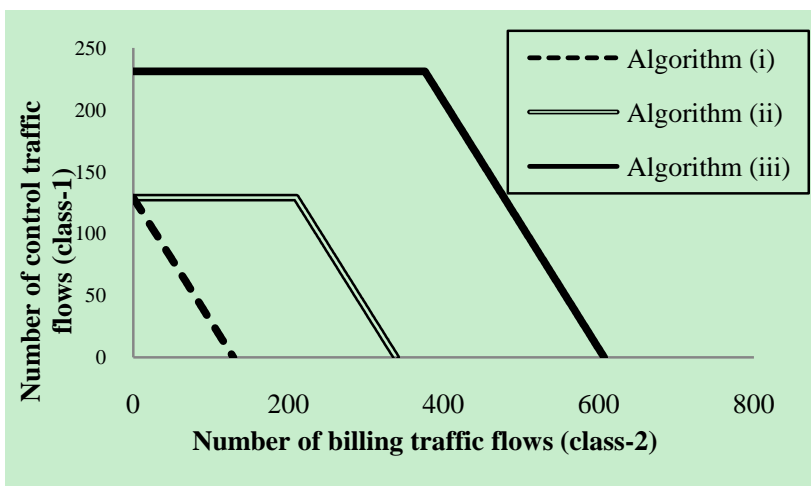

(a)

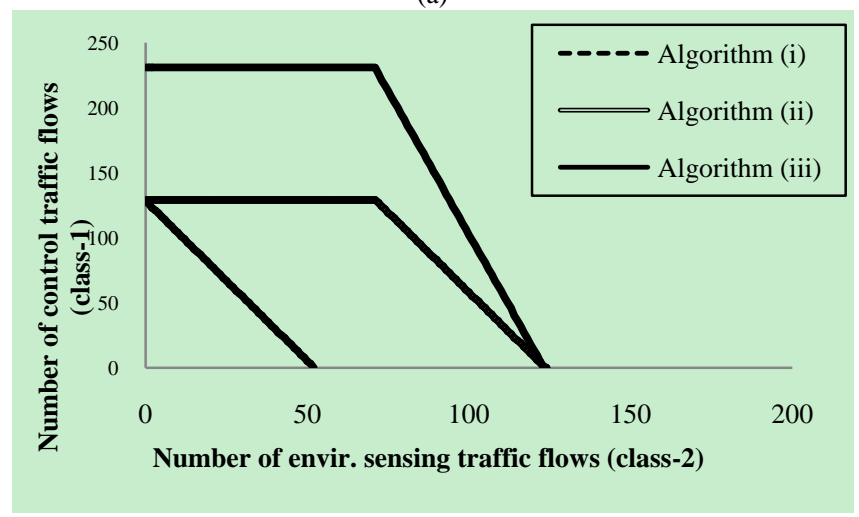

(b)

Fig. 9. Simulated call admissible regions of class- 1 and class- 2 traffic being (a) control traffic and billing/usage traffic (b) control traffic and environmental sensing traffic, respectively.

Fig. 9 gives the simulation results of three algorithms in two scenarios. The three algorithms are i) FCFS scheduler w/o packet concatenation at AL, ii) deadline ordered scheduler w/o packet concatenation at AL, and iii) deadline ordered scheduler w/ packet concatenation at AL. Note that all three algorithms adopted packet concatenation at RLC layer according to the LTE standard. As shown in both Fig. (a) and (b), it is obvious that the call admissible region of dead-line ordered scheduler is larger than that of FCFS scheduler. The CAC region of FCFS case only exhibits roll-off trend with no flat part, because FCFS scheduler has to transmit theclass-2 flow first when all class-2 sub-packets enter the DOQ earlier than the class-1 sub-packets. Thus, the number of class- 1 flows decreasesas $n_{2}$ increases from 0 .As compared with Algorithms ii) and iii), it is observed that the employment of packet concatenation at $\mathrm{AL}$ significantly improves the bandwidth efficiency. However, in Fig. (b),the slope of the roll-off lines of Alg3 becomes larger than that in Fig. (a) (i.e., inferior performance), because the lager the payload size, the smaller the ratio of header size to payload size, and the less benefit that concatenation can bring. The worst case occurs at the Alg3 in Fig. (b). Since the maximum number of class- 2 sub-packets that can be concatenated in one AL payload of a LTE packet is one (i.e., the integral part of the ratio of $L_{\max }$ to the size of class-2 sub-packet is equal to 1), after all packets containing class-1 sub-packet are transmitted, every packet can only contain one class- 2 sub-packet, i.e., we cannot concatenate any class-2sub-packet into a AL payload of a LTE packet. As a result, the descending curve of $\operatorname{Alg} 3$ and $A \lg 2$ intersect with each other at the bottom where $n_{1}=0$, i.e., Alg 3 and $\operatorname{Alg} 2$ have the same performance when $n_{1}=0$.

\section{CONCLUSION}

We have illustrated a concentrator design with deadline-ordered scheduler and packet concatenation at the application layer to tackle the high protocol overhead issue in smart grids. In addition, when the concentrators are assigned a fixed amount of bandwidth, the CAC can be obtained to ensure the delay guarantees of the smart meter real-time application. From the simulation results, the employment of deadline ordered scheduler and packet concatenation at application layer is shown capable of significantly improving the channel capacity and thus has a larger call admissible region. In addition, based on the CAC procedure demonstrated in this work, one can easily estimate the capacity of the smart grid wireless backhaul and determine the maximal number of nodes that can be supported by the smart grid network without violating their delay constraint.

It is observed that the call admissible region attained by the analytical method is smaller than that by simulation since strict mathematic derivation need to be followed which leads to additional overhead. Hence, we believe that the call admissible region obtained by simulation is closer to the true capacity. However, the analytical result does provide a quick approach to estimate the capacity of the smart grid wireless backhaul.

\section{ACKNOWLEDGMENT}

This work was supported by the National Science Council, ROC, under grants NSC 100-2221-E-002-186-MY2.

\section{REFERENCES}

[1] IEEE smart grid. [Online]. Available: http://smartgrid.ieee.org/

[2] D. Niyato, L. Xiao, and P. Wang, "Machine-to-machine communications for home energy management system in smart grid," IEEE Comm. Magazine, vol. 49, no. 4, pp. 53-59, Apr. 2011.

[3] 3GPP Standard36.213, Evolved Universal Terrestrial Radio Access (E-UTRA)-Physical Layer Procedures.

[4] R. H. Khan and J. Y. Khan, "A comprehensive review of the application characteristics and traffic requirement sofa smart grid communications network," Computer Networks, vol. 57, pp. 825-845, Feb. 2013 
[5] Y. Xu and W. Wang, "Wireless mesh network in smart grid: modeling and analysis for time critical communications," IEEE Trans. Wireless Communication, vol. 12, no. 7, July 2013.

[6] W. Wang, S. C. Liew, and V. O. K. Li, "Solutions to performance problems in VoIP over a 802.11 wireless LAN," IEEE Trans. Vehicular Technology, vol. 54, no. 1, pp. 366-383, Jan. 2005.

[7] W. C. Hong and Z. Tsai, "A multichannel scheduler for high-speed wireless backhaul links with packet concatenation," IEEE Trans. on Mobile Computing, vol. 9, no. 2, pp. 201-214, Feb. 2010.

[8] N. R. Figueira and J. Pasquale, "A schedulability condition for deadline-ordered service disciplines," IEEE/ACM Trans. Networking, vol. 5, no. 2, pp. 232-244, Apr. 1997.

[9] Z. Alliance. (2008). ZigBee Specification. [Online]. Available: http://www.zigbee.org/Specifications.aspx

[10] L. Yonge, J. Abad, K. Afkhamie, L. Guerrieri et al., "An overview of the homeplugAV2 technology," Journal of Electrical and Computer Engineering, vol. 2013, pp. 1-20, 2013.

[11] C. Ergen. ZigBee/IEEE 802.15.4 Summary. [Online]. Available: http://www.eecs.berkeley.edu/csinem/academic/publications/zigbee.p df

[12] H. A. Latchman, S. Kater, L. Yonge, and S. Gavette, Homeplug AV and IEEE 1901: A Handbook for PLC Designers and Users, Wiley-IEEE Press, March 2013.

[13] D. Ferrari and D. Verma, "A scheme for real-time channel establishment in wide area networks," IEEE J. Selected Areas in Comm, vol. 8, no. 3, pp. 368-379, Apr. 1990.

[14] J.-T. L. Boudezc and P. Thiran, G Systems for the Internet, Springer-Verlag, 2004.

[15] F. Aalamifar, H. Hassanein, and G. Takahara, "Viability of powerline communication for the smart grid," in Proc. 26th Biennial Symposium on Communications (QBSC), Kingston, 28-29 May 2012.

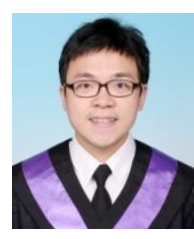

Ting-Chu Lee received the B.S. degree in electrical engineering from National Tsing Hua University (NTHU), Hsinchu, in 2012. He is currently working toward the master degree at the Graduate Institute of Communication Engineering, National Taiwan University, under the guidance of professor Zsehong Tsai. His research interests include wireless network protocol and next generation wireless network.

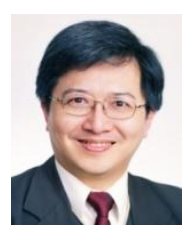

Zsehong Tsai received the B.S. degree in electrical engineering from National Taiwan University (NTU), Taipei, in 1983, and the M.S. and Ph.D. degrees from the University of California, Los Angeles, in 1985 and 1988, respectively. During 1988-1990, he worked as a member of Technical Staff at AT\&T Bell Laboratories, where he investigated performance aspects of network management systems. Since 1990, he has been with the Department of Electrical Engineering and Graduate Instate of Communication Engineering of NTU, where he is currently a professor. He has been active in telecommunication deregulations since Taiwan started the liberalization process of its telecom market. In 2000, he served as a cochair of the 3G Study Group for DGT, the telecommunication regulator in Taiwan. Since 2004, he has also been an independent director of Chunghwa Telecom, Co. Dr. Tsai's research interests include broadband Internet, next generation wireless network, telecomm regulations, and digital convergence policy. Dr. Tsai is a receipt of the CIE (Chinese Institute of Engineers) Technical Paper Award in 1997. He is also a member of IEEE. 